\section{A Comparison of Photomicrographs Imaged Through a Late 18th $C$. Thomas Ribright, Cuff-Type, Brass Microscope and a Modern Olympus Optical Microscope}

\author{
By D.Jones* and J.Reid. ${ }^{* *}$
}

${ }^{*}$ Retired Research Microbiologist Aberdeen

**School of Physics, The University, Aberdeen, Scotland

\section{Introduction}

In 1967,Bradbury (I) published a detailed study of the optical properties of early, compound microscopes using modern photo micrographic techniques. This was followed by Bracegirdle's studies(2) on the performance of 17th and 18th C. microscopes and later by Jones's comparative study of a Victorian microscope with an 18th C. Cuff-type microscope, signed 'Dollond' (3).0n the suggestion of the late Dr. S. Bradbury (personal communication), the present study on the assessment of a Ribright microscope includes photomicrographs of specimens in slides similar to the ones he used, i.e. a blow-fly proboscis and diatoms.
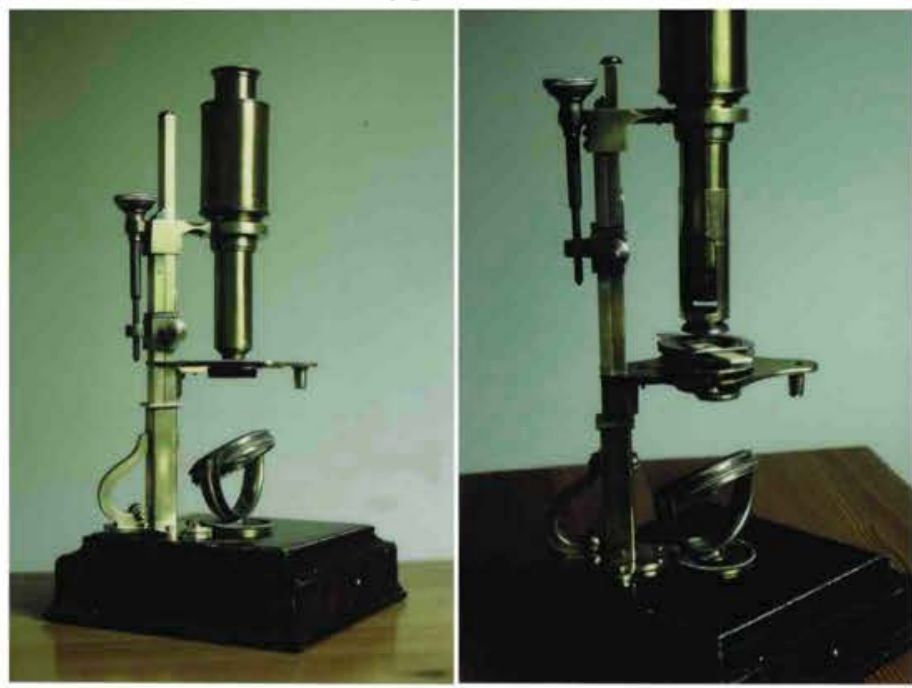

Figure 1. Cuff-type microscope, signed 'Thomas Ribright London fecit'. Figure 2. As Fig. 1, but fitted with lieberkuhn holder and lieberkuhn.

\section{Materials and Methods}

\section{The Microscope}

The instrument designed by Cuff in the 18th Century was notable because it was made of brass, incorporated a stage that allowed easy access to the specimen being examined, and was one of the first microscopes to feature a fine focusing facility(4).The microscope described here (Figure 1) is a typical Cuff-type instrument, signed on the stage with an engraving 'Thomas Ribright London fecit,' and purchased at Auction in Christie's, South Kensington, London. The body tube $(17.5 \mathrm{~cm}$ long, including eyepiece) fits into an arm of the movable back section of the split vertical pillar support. This back section can be secured to the front, fixed section of the pillar by means of a clamping screw. Coarse focusing is achieved by raising or lowering the back section of the split pillar, fine focusing is also achieved through this section but by rotating the horizontal screw. The eared stage, which has a figure 5 etched on its undersurface, is screwed to the front section of the pillar, and has a socket for a condenser lens and an aperture for a stage forceps or fish/frog pan.

The fixed, front section of the pillar is marked on the side with lines and figures 1-6 to correspond with the six numbered objectives ( 1 being the highest magnification lens, 6 the lowest). Thus, the movable back section of the pillar can be adjusted to the height appropriate to the lens being used. The long nose of the body tube has etched lines numbered 1-6
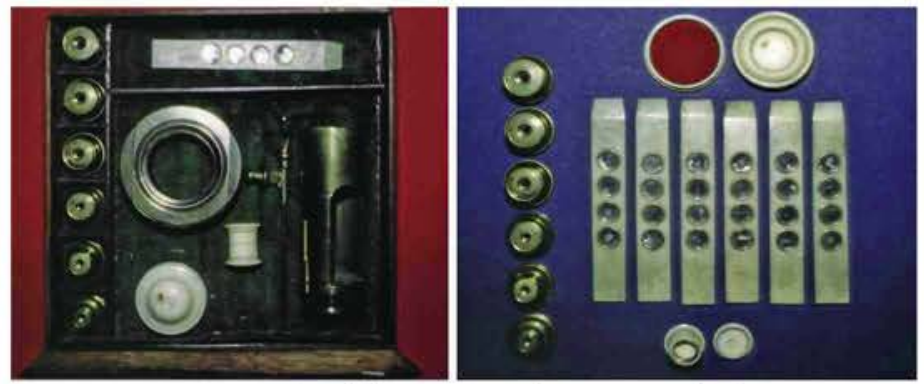

Figure 3. Baize-lined draw with 6 objectives,circular brass spring tage, stage forceps, ivory sliders, ivory box with talcs and split brass rings, lieberkuhn holder and circular ivory box with lieberkuhn.

Figure 4. Accessories in more detail.
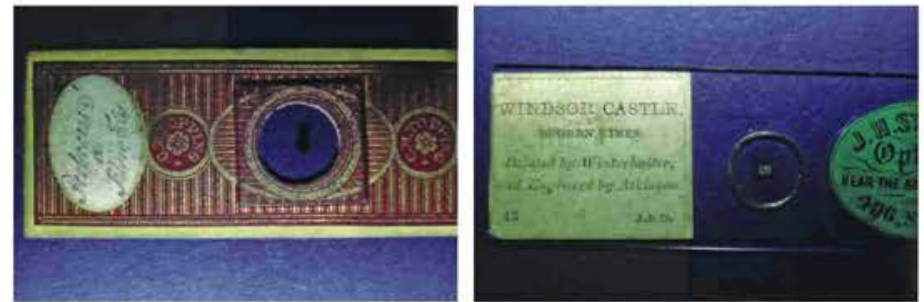

Figure 5. Blow-fly proboscis (feeding organ) mounted on Victorian glass slide.

Figure 6. J. B. Dancer slide with microphotograph of 'Windsor Castle,

Modern Times'.

( 1 at the top, 6 at the bottom) to position the brass sleeve, on to which the lieberkuhn is screwed, for each of the appropriately numbered objectives (Figure 2).There is a circular slider holder, or spring stage, and a concave sub-stage mirror. The pillar is secured to a brass plate that is screwed to the veneered mahogany-oak plinth base fitted with a draw ( $13 \mathrm{~cm}$ wide) lined with green baize (Figure 3 ). The plinth base has a number 7 pencilled on the interior, which could be the batch number of the joiner, according to Turner(4).The eye-piece, which has two lenses, screws into the body tube. There are six objectives, each with a biconvex lens, and six ivory sliders with various specimens, which include fish scales, lice and insect limbs (Figure 4). Accessories also include a tubular ivory box with talcs (discs of mica to secure specimens in the sliders) and split brass rings (to secure the talcs in the sliders), and a flattened, circular, ivory box (with a screw-on lid) for the lieberkuhn. The microscope and accessories are contained in the original (according to the Sale Catalogue) pyramidshaped oak box with a drawer for the accessories.
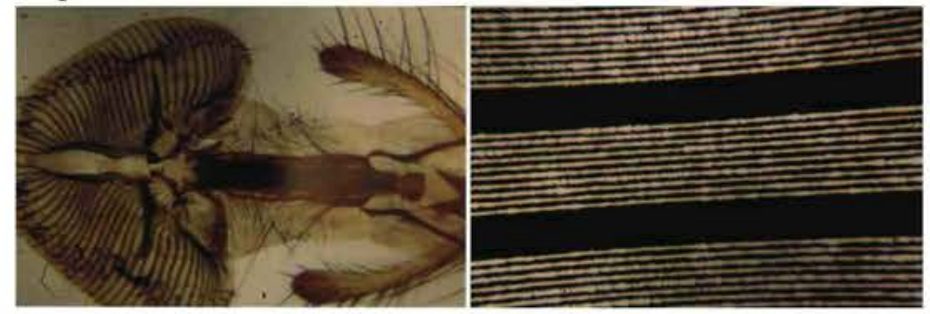

Figure 7. Blow-fly proboscis. Lens 4, Ribright microscope. Actual width of proboscis $=1.8 \mathrm{~mm}$.

Figure 8. Abbe Test slide.Lens 4, Ribright microscope.

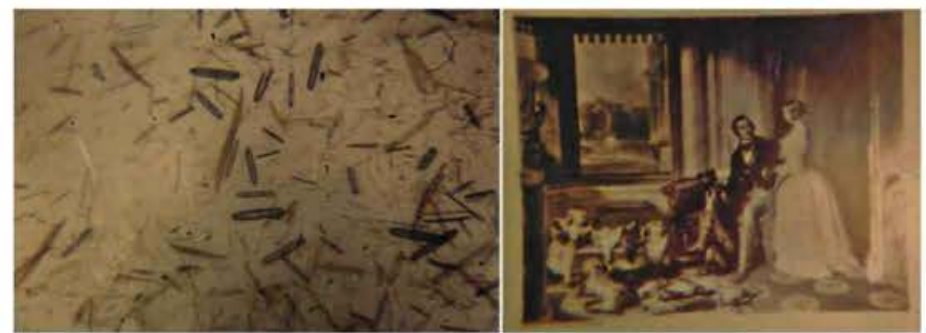

Figure 9. Diatoms. Lens 4, Ribright microscope.

Figure 10. J. B. Dancer slide. Lens 4, Ribright microscope. Actual width of microphotograph $=1.6 \mathrm{~mm}$. 
Gesham Scientific
Instruments is changing...

In July 2005 after 10 successful years in the X-ray analytical market, Gresham was purchased by e2v technologies.

\section{As part of e $2 v$ we are expanding our horizons by:}

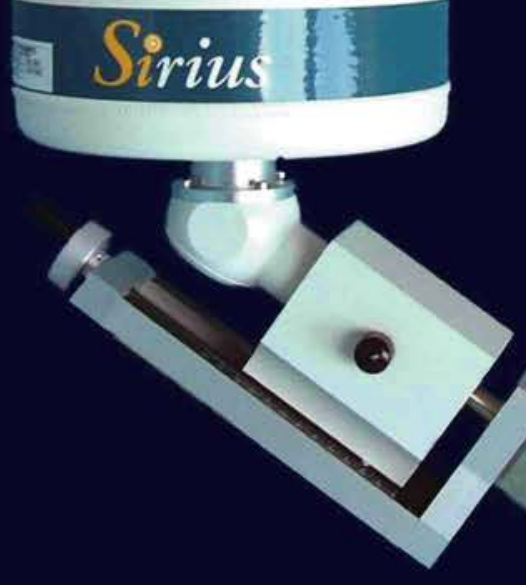

Changing our name to e2v scientific instruments

A new name but the same specialist team, bringing innovations in technology and increased levels of customer support worldwide

Introducing a new US-based EDX detector repair and upgrade facility

As the world's leading independent EDX detector company we are bringing our wealth of experience directly to the United States through our New York office

- Detectors from any manufacturer repaired or upgraded

- Fast and reliable turn-around

- Competitively priced

Providing a comprehensive product range, including:

- 'Sirius' High Performance EDX detectors for SEM and TEM

- 'Titan' Digital Pulse Processing Technology

- Specialised XRF detectors

- Detector Repairs and Upgrades
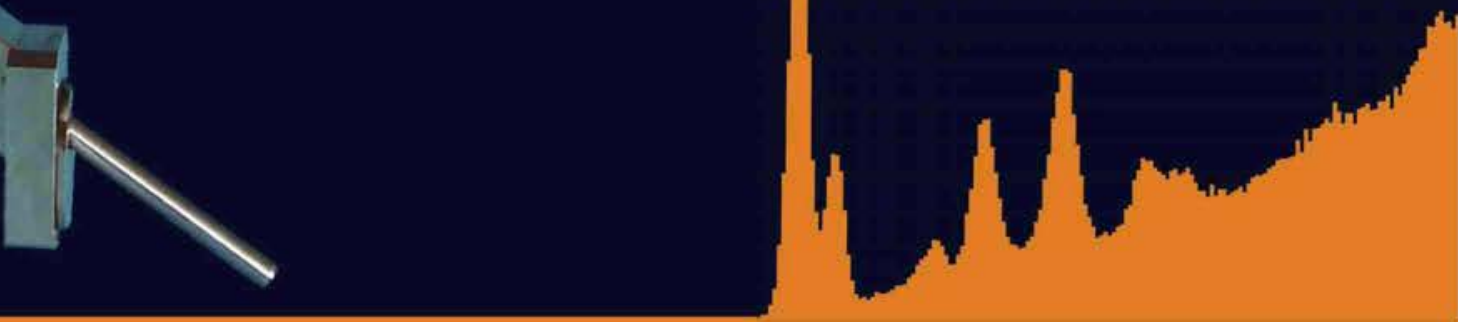

e2v scientific instruments...

bringing world class sensor technology to the X-ray analytical market.

To find out more about our products

or new facilities please visit:

www.e2vsi.com

e2v

email:e2vsi@e2v.com 


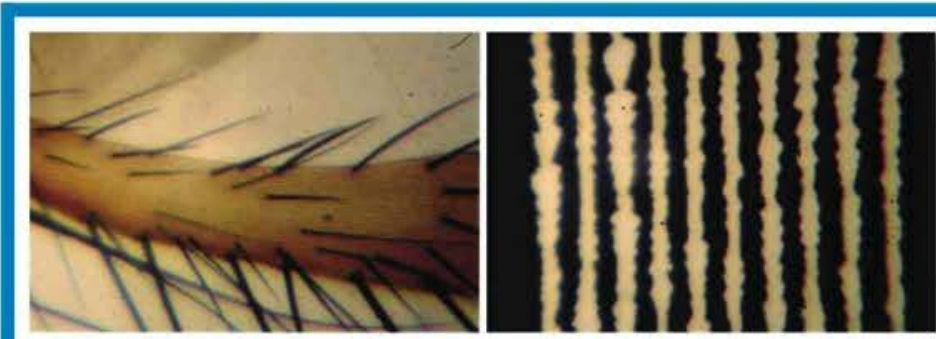

Figure 11. Blow-fly mouth-part palp. Lens 2, Ribright microscope. Figure 12. Abbe Test slide. Lens 2, Ribright microscope.
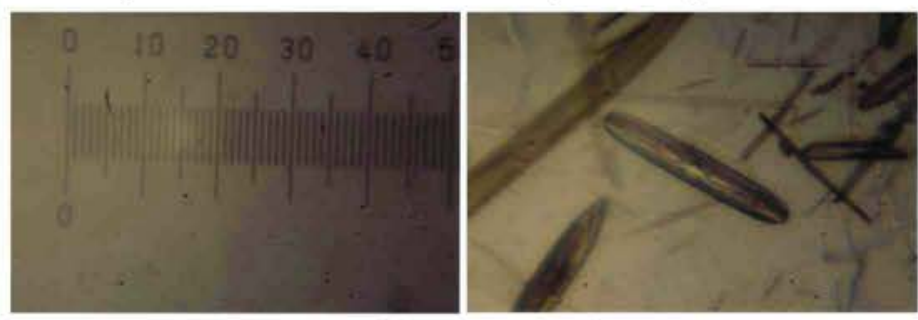

Figure 13. Micrometre slide. Lens 2, Ribright microscope.

Figure 14. Diatoms. Lens 2, Ribright microscope. Actual length of diatom at centre of field $=0.27 \mathrm{~mm}$.

\section{Authenticity of the Ribright microscope}

The question of authenticity arises when any antique is being assessed. The instrument described here was purchased at a reputable London auction house and this ensures that the microscope has been examined by an expert and that the description in the sale catalogue is accurate. For example, the instrument and accessories are described as being 'in the original pyramid-shaped oak case.' The fact that the six, numbered objectives screw into the body tube is indicative that they were likely to have been made for this microscope only, because threads were individually hand-made until a standard thread size was introduced by the Royal Microscopical Society in the 19th Century (Proc. R. Microscop.
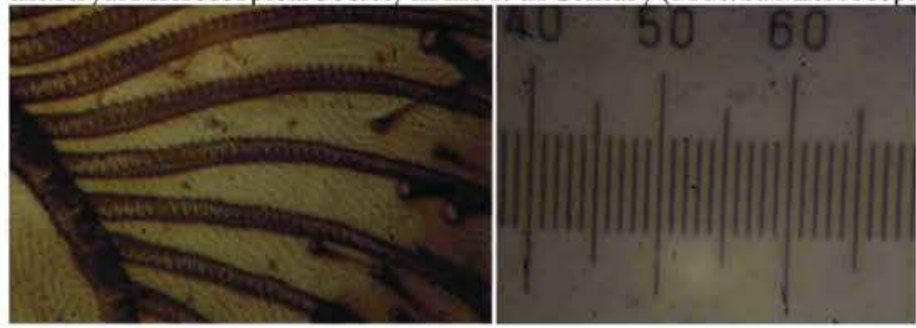

Figure 15. Blow-fly proboscis pseudotracheae. Lens 1, Ribright microscope. Horizontal field width $=0.3 \mathrm{~mm}$.

Figure 16. Micrometre P4 slide. Lens 1, Ribright microscope. Each division 10 micrometres.

Soc. 21,$163 ; 1986)$. Similarly, the lieberkuhn screws onto the lieberkuhn holder that itself fits neatly onto the body tube and can be adjusted according to the objective used. None of these items can be interchanged with the accessories that accompany another Cuff-type microscope (signed 'Dollond London') in the author's collection. All important too is the fact that the microscope described here is signed on the stage with the name and location of a well known 18th-19th Century maker of scientific instruments.

\section{Slides}

Photographic images were made with objectives $1,2,4$ and 5 to test the optical properties of their lenses.

Slide 1. Is an example of an English commercial glass microscope slide (1850-70), which is $7.5 \mathrm{~cm}$ by $2.5 \mathrm{~cm}$ (Figure 5 ) and typically bound with red coloured and patterned paper (7).It has the name 'C.M. TOPPING' printed twice on the paper covering and 'Proboscis of a Blow Fly' is written on an oval-shaped paper label at one end of the slide.

Slide 2. This is a John Benjamin Dancer glass slide (Figure 6) of the same dimensions as the above slide and also from the Victorian Period.
It has a mounted microphotograph of the Prince Regent and Queen Victoria and is entitled 'Windsor Castle, Modern Times.' Printed on the slide is 'Painted by Winterhalter and engraved by Atkinson' but according to Bracegirdle and McCormick (8) the painting is by Landseer. The slide is numbered ' 43 ' and has the initials 'J.B.D.' Printed on a green oval-shaped disc of paper on the slide is 'J. H.Steward, Optician, near Adelphi Theatre, 406, Strand'.

A similar type Victorian glass slide (signed J.B.D.), but with a microphotograph entitled 'Trafalgar Square, Charing Cross' and initials 'A.R.' was imaged through objective lens 5 . Not imaged were two accompanying Victorian slides of microphotographs and also with the initials 'A.R.'

Slide 3. An Abbe Test slide (Carl Zeiss, Jena) that was used to test for spherical aberration; each band of 10 lines on this slide is $0.6 \mathrm{~mm}$ wide. This is a modern slide made by Zeiss.
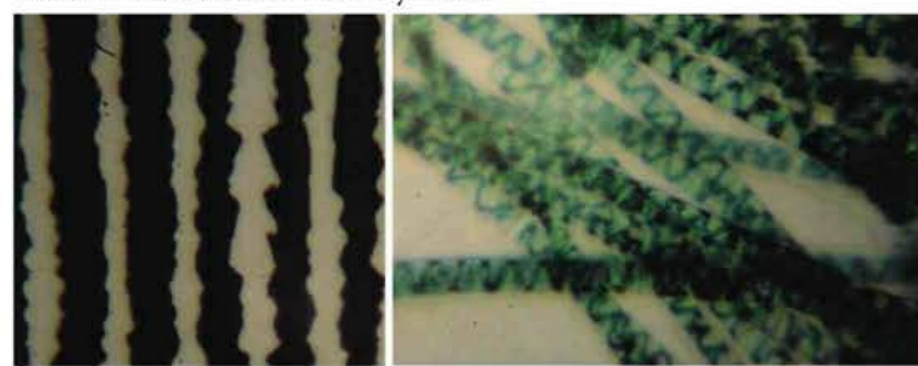

Figure 17. Abbe Test slide. Lens 1, Ribright microscope.

Figure 18. Spirogyra sp.:spirally-coiled chloroplasts. Lens 1, Ribright microscope. Actual width of algal filaments $=18 \mu \mathrm{m}$. approx.

Slide 4. A stage micrometre slide (Agar Scientific), displaying a $1 \mathrm{~mm}$ scale divided by lines into 10 micrometre sections, was used to indicate the dimensions of the specimens examined.

Slide 5 . This is a special diatom slide (No.1717) made recently by Bernard Hartley, Somerset. It is a combined sample of diatoms, which includes Amphipleura pelluda, Pleurosigma angulatum, and Stauroneis phoenicenteron and other species. Diatoms were viewed in the last Century to check the quality of condensers and objectives(9).

Slide 6. This slide of the green alga, Spirogyra sp. stained with Malachite green, was prepared by Mr. J. Wells.

Another item included in the Ribright accessories is a large stained, wooden slide, $6.7 \times 2.0 \times 0.25$ inches thick, in which are separately mounted under 1 inch diameter glass slips, secured with split metal rings, two insects-Alder Beetle (numbered 503)and Forficula auricularia (504), which is commonly known as the earwig. According to Turner(7) these large wooden slides were introduced towards the end of the 18th Century for use with solar and lucernal microscopes.

With the exception of the wooden and ivory sliders, the other(glass)slides are obviously not original to the microscope but have been added later.

\section{Photomicrography}

An Olympus OM10 single reflex $35 \mathrm{~mm}$ camera, with lens removed
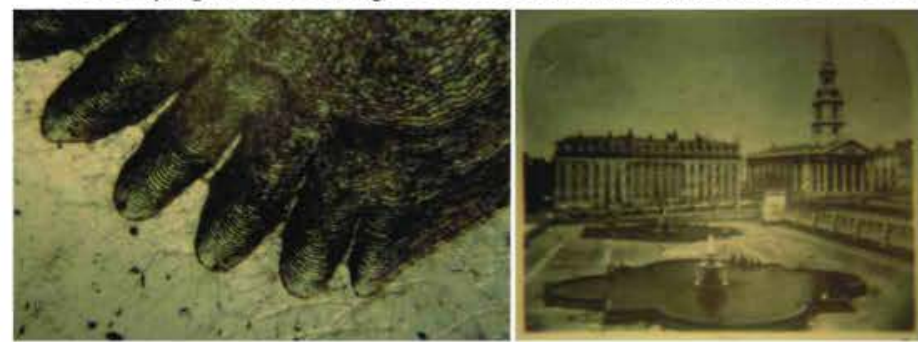

Figure 19. Anterior end of Perch scale in ivory slider, imaged with objective lens 5 of the Ribright microscope. Horizontal width of the image $=2 \mathrm{~mm}$.

Figure 20. Microphotograph of Trafalgar Square imaged through lens 5 of the Ribright microscope. Scale as for figure 10. 


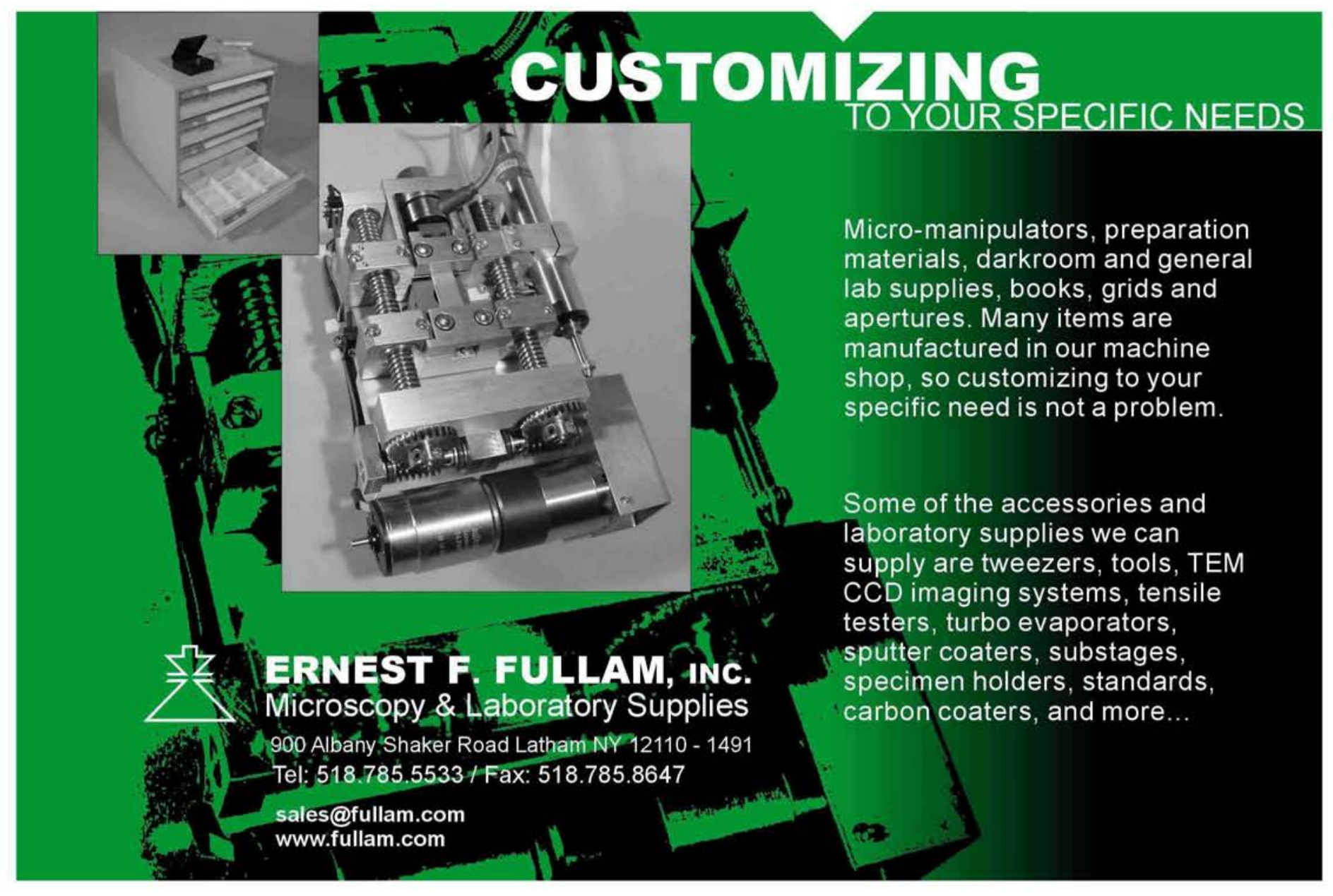




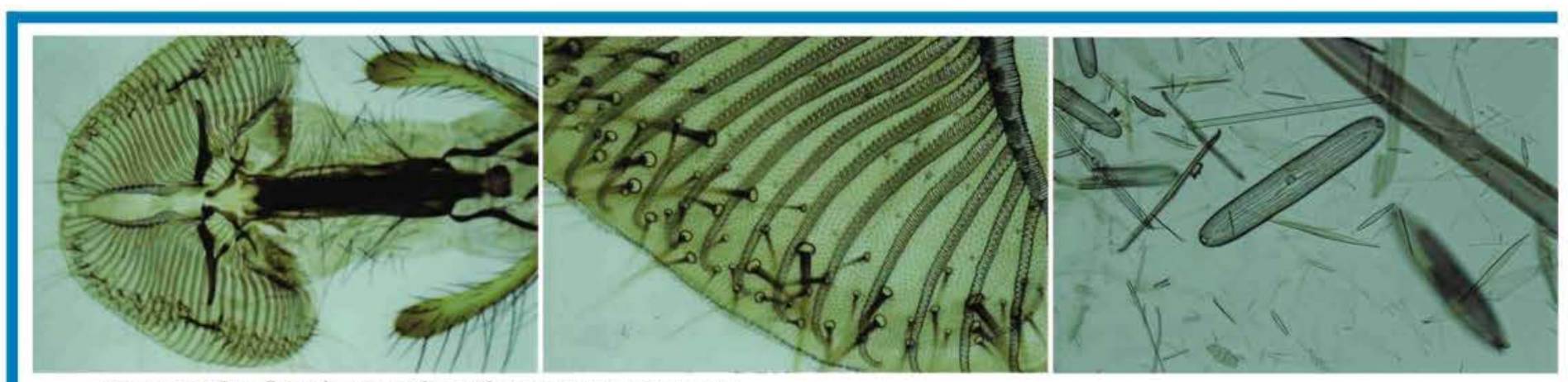

Figure 21. Blow-fly proboscis, $\times 4$ lens, Olympus Provis microscope.

Figure 22. Blow-fly proboscis: pseudotracheae $\times 10$ lens, Olympus Provis microscope.

Figure 23. Diatoms, $\times 10$ lens, Olympus Provis microscope.

and two extension tubes $(20 \mathrm{~mm}$ and $12 \mathrm{~mm})$ attached, was fitted to the eyepiece of the microscope and held in place with a tripod stand; the source of light was a 60 watt tungsten electric light bulb in an anglepoise lamp stand. Fujichrome Professional 64T colour film was used to record images.

\section{Results}

\section{Slides imaged through the Ribright microscope}

Calliphora vomitoria (Order Diptera) is the bluebottle (blowfly) seen in houses during the summer months (10). Figure 7 features the labium, which is the feeding organ, or proboscis, of this non-blood sucking insect, imaged through lens 4 . It has two lobes, or labella, with pseudotracheae that are the tubes for 'mopping up' surface liquids. Also featured in Figure 7 are two palps, which are components of the mouthparts. All these structures are well resolved by lens 4 but there are clear signs of spherical aberration when the Abbe test slide is imaged and this is manifested by the curvature of the lines towards the periphery of field of view (Figure 8 ).The diatoms are not particularly well resolved with lens 4 (Figure 9).

The microphotograph of Landseer's painting,although somewhat blurred when viewed through lens 4 ,still reveals the main features (Figure 10). Figure 11 again illustrates a mouth-part palp, but imaged through lens 2. Remarkable detail is seen, without much evidence of spherical aberration. This is confirmed when the Abbe test slide is imaged with the same lens (Figure 12).However, there is some distortion of the etched lines on the micrometre slide (Figure 13)and particularly towards the periphery of the image. Resolution of the diatoms shell markings was not achieved with this lens (Figure 14).

Good resolution was achieved with lens 1 and the pseudotracheae are particularly clear (Figure 15) but there is some evidence of peripheral distortion of the micrometre slide divisions (Figure 16). This is not obvious when the Abbe test slide is viewed (Figure 17). Good resolution of Spirogyra spirally-coiled chloroplasts and cross-walls was achieved with this lens (Figure 18).

Similar images to those recorded through lens 4 were obtained with lens 5 , which was also used to photograph fish scales in one of the sliders accompanying the Ribright microscope (Figure 19) and a microphotograph of Trafalgar Square in the Victorian slide (Figure 20).

\section{Specimens imaged through a modern microscope}

For comparison, the blow-fly proboscis and diatoms were imaged through an Olympus optical Provis microscope fitted with a dedicated automatic camera system. The objectives were Universal Plan Apochromats. The low magnification $(\times 4$ lens, N. A. 0.16 , working distance $13 \mathrm{~mm}$ ) micrograph of the proboscis( Figure 21) did not differ markedly in detail from that imaged through the Ribright lens 4 (Figure 7 ) with a N. A. of 0.04 , but the fine structure of the pseudotracheae (Figure 22) was far better resolved with the Provis $\times 10$ lens(with a N. A. aperture of 0.4 and working distance of $3.1 \mathrm{~mm}$ ) than with the Ribright lens 1 (Figure 15) with a N. A. of 0.13. Excellent resolution of the fine structure of the silica frustules (shells) of the diatoms was achieved with the Provis

$\times 10$ lens(Figure 23 ) in contrast with the poor image produced with the Ribright lens 2 (Figure 14) with a N. A. of 0.12 .

\section{Optical properties of the Ribright lenses}

The focal lengths $(\mathrm{mm})$ of the objective lenses 1-6,calculated from measurements made on an optical bench,are 4.3, 6.0, 12.3, 23.5, 23.35, and 30.24 respectively.The numerical apertures of the same lenses $1-6$ (radius of internal aperture in $\mathrm{mm}$, divided by the focal length) are 0.13 , $0.12,0.06,0.04,0.05$, and 0.03 respectively. The numerical aperture $(\mathrm{N}$. A.) is the measure of the resolving power of the lens, the higher the $\mathrm{N}$. A. , the better the resolution. The focal length $(\mathrm{mm})$ of the eye lens, one of the two lenses (the other being the field lens) in the eye-piece, is 18.4. The focal lengths of the objective lenses 4 and 5 are close ( 23.5 and 23.35) and this suggests that lens 4 maybe a replacement lens. This is because both lenses require the arm, holding the microscope tube to be adjusted to focus line 5 on the front fixed pillar to give approximate focussing of specimens on the stage (without the Bonanni spring stage in position).

\section{Conclusion}

A full assessment has been made of an example of a Cuff-type compound microscope made in the late 18th Century. The accessories are described, some of which do not belong to the microscope. The lenses are assessed and their optical performance judged. One objective lens is clearly not one of the set of six, even though the objective case can be readily screwed onto the body tube.

\section{REFERENCES}

1. Bradbury, $\mathrm{S}$. The quality of the image produced by the compound microscope: 1770-1840, pp 151-173. In: Historical Aspects of Microscopy (ed. S. Bradbury \& G. L. 'E. Turner).Heffer, Cambridge, England, 1967.

2. Bracegirdle, B. The performance of seventeenth-and eighteenth-century microscopes. Medical History 22, 187-195, 1978.

3. Jones, D., 18th and 19th century microscopes reappraised. Microscopy and Analysis 64 (U.K. edit.), 9-11, 1998.

4. Turner, G. L.'E. The Great Age of the Microscope. Adam Hilger, Bristol and New York,1989.

5. Directory of British Scientific Instruments Makers 1550-1850. Zwemmer. Christie’s Cat. 125, 1995.

6. Calvert, H. R. Scientific Trade Cards in the Science Museum's Collection(London), 1971.

7. Turner, G. L. 'E. Collecting Microscopes. Cassell Ltd. London, Sydney, Johannesburg, 1981.

8. Bracegirdle, B. and McCormick, J. B. The Microscopic Photographs of J.B.Dancer. Science Heritage Limited, Chicago,Illinois, 1993.

9. Hartley, W. E. The Light Microscope. Its Use and Development. Senecio Publishing Company,Oxford,1993.

10. Chinery, M. Insects of Britain \& Northern Europe. Harper Collins, London, New York, Sydney,1994.

\section{Acknowledgements}

We thank the late Dr.Savile Bradbury for reading the manuscript and making useful comments to improve it. The Provis photomicrographs are courtesy of Mr. Alan Lesslett, Olympus Optical, and we express our appreciation for his help and discussions regarding this study. 

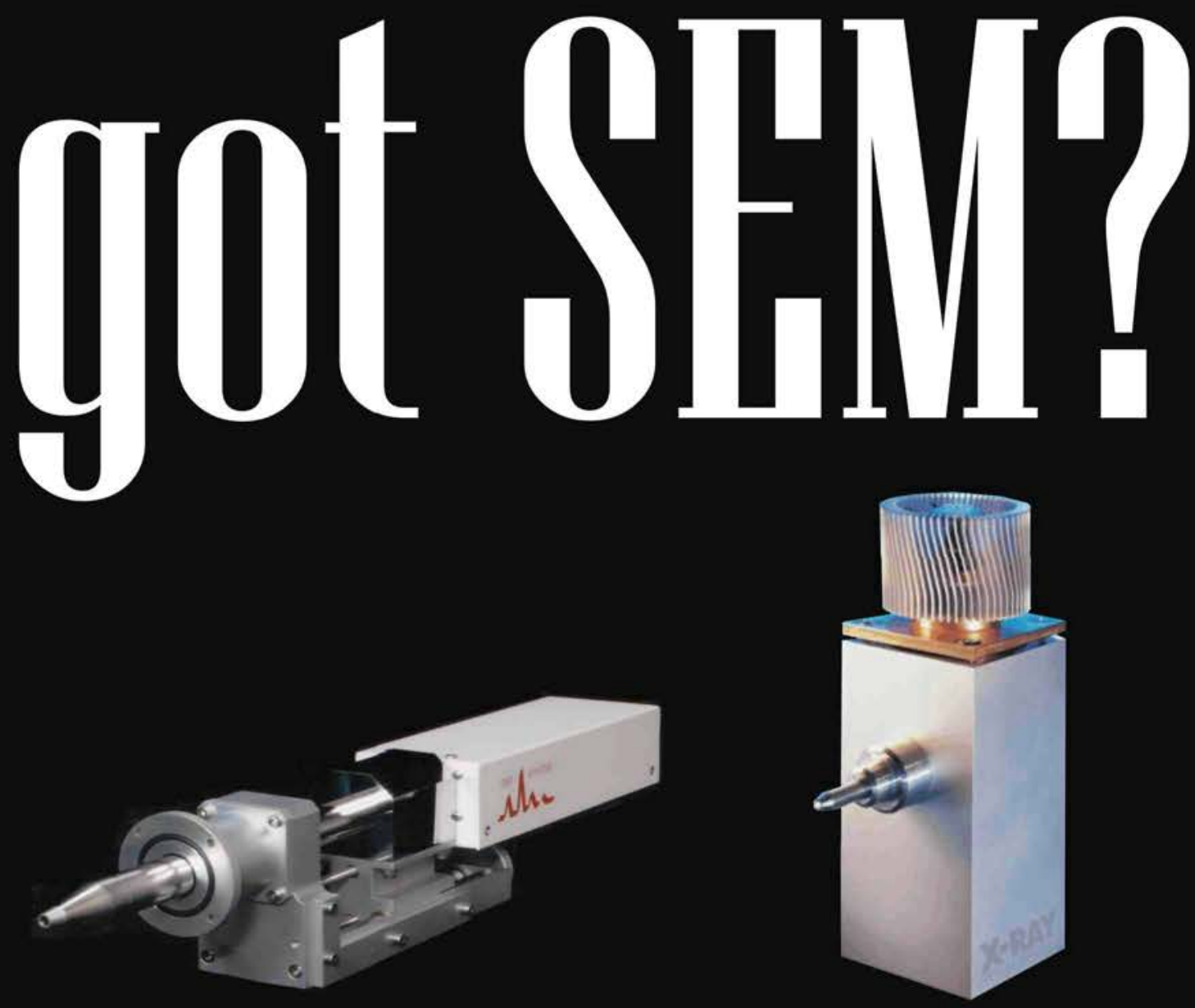

Add MicroX-ray Fluorescence to any Scanning Electron Microscope.

Complements:
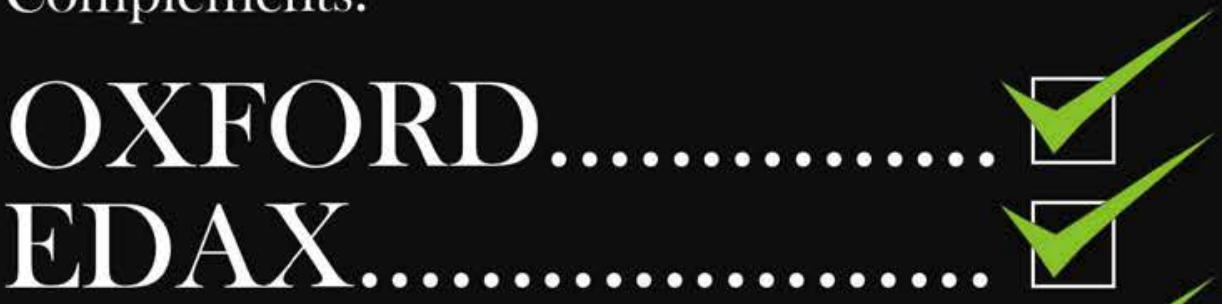

NORAN.................

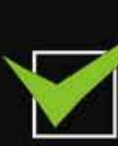

$D\left(\frac{1}{2}\right)$

\section{IXRF SYSTEMS}

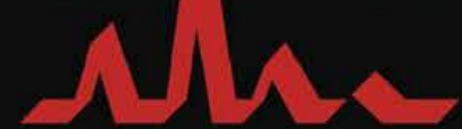

